\title{
Optical multiplexed bioassays on photonic crystals for breast cancer biomarker detection
}

\author{
Tommaso Pileri ${ }^{1 *}$, Alberto Sinibaldi ${ }^{1}$, Agostino Occhicone $^{1}$, Elena Giordani $^{2}$, Matteo Allegretti ${ }^{2}$, Peter Munzert $^{3}$, \\ Frank Sonntag ${ }^{4}$, Norbert Danz ${ }^{3}$, Patrizio Giacomini ${ }^{2}$ and Francesco Michelotti ${ }^{1}$ \\ ${ }^{1}$ Department of Basic and Applied Science for Engineering, Sapienza University of Rome, Via A. Scarpa 16, 00161, Rome, Italy. \\ ${ }^{2}$ IRCCS Regina Elena National Cancer Institute, Via Elio Chianesi 53, 00144 Rome, Italy. \\ ${ }^{3}$ Fraunhofer Institute for Applied Optics and Engineering IOF, A. Einstein-Str. 7, 07745 Jena, Germany. \\ ${ }^{4}$ Fraunhofer Institute for Material and Beam Technology IWS, Winterberg-Str. 28, 01277 Dresden, Germany.
}

\begin{abstract}
An optical biosensor for proteomic breast cancer biomarker detection in complex media is presented. Bloch Surface Waves (BSW) excited onto one dimensional photonic crystal (1DPC) were used to probe the interaction of HER2 with three antibody species and an inert protein (Bovine Serum Albumin BSA). The optical system combines Label-Free readings to track the bioassay real-time development and Fluorescence emission quantification to evaluate the level of specific interaction between the antigen and the antibodies. The results confirm a distinguishable level of affinity between the antibodies and the analyte according to their specificity even at low antibody surface density (about $1173 \mathrm{pg} / \mathrm{mm}^{2}$ ).
\end{abstract}

\section{Introduction}

Non-invasive diagnostics via cancer biomarker detection is a constantly increasing focus of research [1]. Among other techniques, optical biosensors based on electromagnetic surface waves represent an opportunity to improve ease-to-use and multiplexing capability while lowering costs and processing time [2]. The first known approach exploiting a spatially confined interaction between a highly enhanced electromagnetic field at the optical interface and the molecular recognition layer, are the surface plasmon polaritons (SPR). However, further significant enhancement of resolution can be obtained by using low lossy all-dielectric multi-layered structures, typically used to excite BSW [3]. This is achieved by combining both Label-Free and Fluorescence investigation of the bioassay occurring over the sensor surface $[4,5]$.

The main purpose of this work is the study of a multiplexed optical biosensor based on BSW able to reveal the presence of the HER2 receptor tyrosine-protein kinase. Altered signalling by epidermal growth factor receptors, like HER2 and other family members, is involved in $20-30 \%$ of all breast cancers [6], representing an appealing subject of investigation in neoplastic transformation and progression. Indeed, these receptors play a critical role because of their involvement in the three main regulation pathways of a cell such as differentiation, growth and proliferation [7].

\section{Methods}

\footnotetext{
* Corresponding author: tommaso.pileri@uniroma1.it
}

\subsection{DPC sensors and optical readout system}

The 1DPC biochips is designed to excite BSW in the visible range when in contact with aqueous solutions. It is composed by two periods of $\mathrm{SiO}_{2}(275 \mathrm{~nm}, \mathrm{n}=1.474+$ $\left.\mathrm{j} 5 \times 10^{-6} @ 670 \mathrm{~nm}\right)$ and $\mathrm{Ta}_{2} \mathrm{O}_{5}(120 \mathrm{~nm}, \mathrm{n}=2.16+$ j5 $\times 10^{-5} @ 670 \mathrm{~nm}$ ) terminated by a topping layer of $\mathrm{TiO}_{2}\left(20 \mathrm{~nm}, \mathrm{n}=2.28+\mathrm{j} 1.8 \times 10^{-3} @ 670 \mathrm{~nm}\right)$ and $\mathrm{SiO}_{2}(20 \mathrm{~nm})$. The final silica layer is deposited to allow for the use of silane based chemical surface functionalization (see next section). For further details on the 1DPC design see ref [8]. The 1DPC deposition process is performed by plasma ion assisted deposition technology (PIAD) onto a prism-shaped plastic (TOPAS) substrate (Fig. 1a-b - insets). This allows to operate in the attenuated total internal reflection configuration (TIR) (Kretschmann-Raether configuration). By using cylindrical lenses, for both modes, the laser beam is focalized on a line along the longitudinal biochip axis [4]. The optical readout system is able to operate in two independent modes.

The first is the Label-Free mode. Using a s-polarized light beam $\left(\lambda_{\mathrm{LF}}=670 \mathrm{~nm}\right)$, the electromagnetic properties evolution due to biorecognition binding events over the surface can be real-time detected. This is performed by tracking the minima position of the reflectivity angular spectra recorded over the entire investigated photonic crystal midline (Fig.1a).

The second operation mode is the Fluorescence mode. This uses a different light source $\left(\lambda_{\text {FLUO }}=\right.$ $635 \mathrm{~nm}$ ) to excite the same surface mode. Sacrificing the excitation efficiency ( $3 \%$ at this wavelength), the present 
design satisfies the BSW coupling condition with the emission peak of the Qdot ${ }^{\mathrm{TM}} 655$ used as fluorescent label in the biorecognition protocol (described in the paragraph 2.2). The dye emitted fluorescence, after being stimulated matching the resonance condition of the optical surface states, is channelled back through the same BSW mode and through the dielectric structure, radiated into a narrow angular range through the TOPAS, and imaged by the CCD (Fig. 1b inset). A dichroic beam splitter separates Label-Free and Fluorescence emission from Fluorescence excitation beam (Fig. 1b inset). For both operation modes, rows and columns of each image recorded by the CCD refers to spectral angular information and lateral position along the biochip midline respectively.
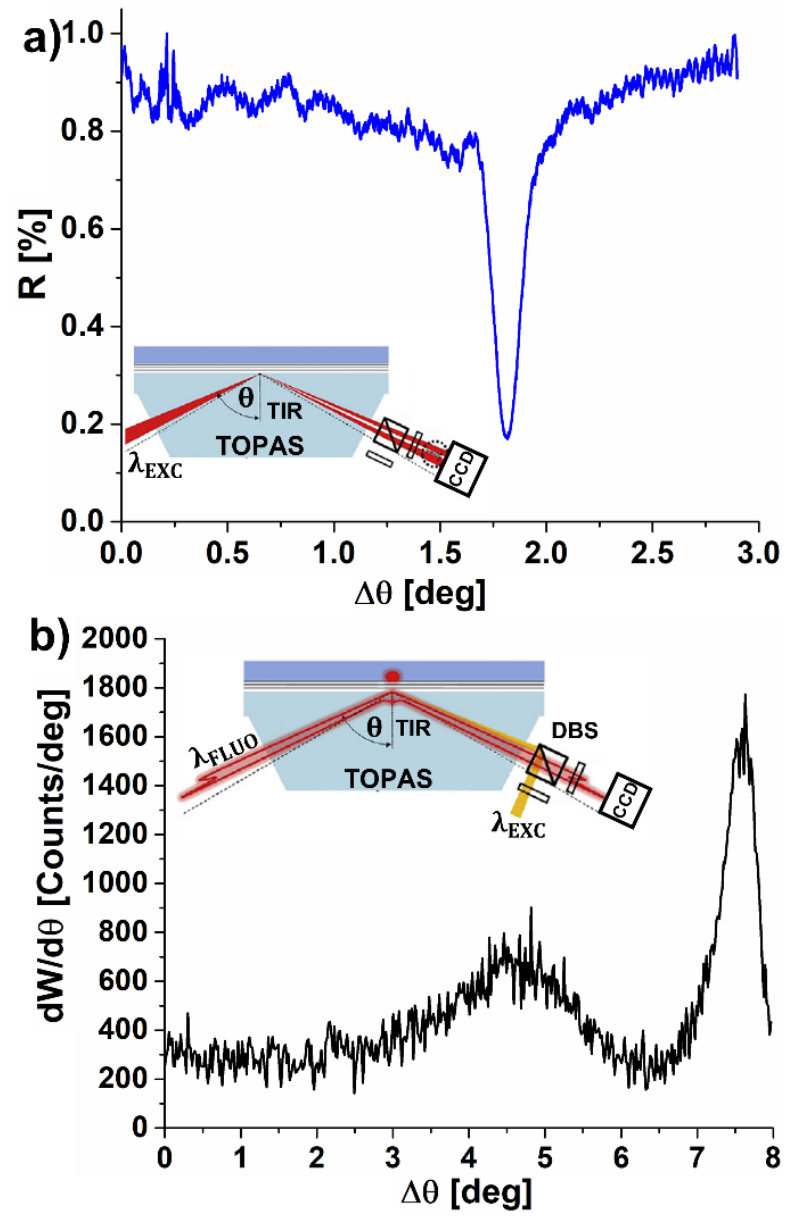

Fig. 1 - The reflectance spectrum shown in (a) has been recorded with Phosphate Buffered Saline $1 \mathrm{x}$ inside the microfluidics (represented by the dark blue layer in the inset). The inset of (a) depicts the light path through the biochip crosssection and light recording system in Label-Free configuration. (b) shows the angular fluorescence intensity emitted by Qdot ${ }^{\mathrm{TM}}$ 655 at the surface of the 1DPC. The inset of (b) shows the fluorescence excitation scheme where the fluorescence emitted is collected with a remarkable directionality.

\subsection{Surface chemistry and bioassay}

In order to covalently bind antibody and proteins onto the 1DPC surface, we silanize the previously piranha cleaned $\mathrm{SiO}_{2}$ thin 1DPC top layer with the (3-aminopropyl)triethoxysilane (APTES) and, subsequently, surface activation with glutaraldehyde is performed [9]. The bio-functionalization step is carried-out by means of a GeSiM Nano-Plotter. The multiplexing characteristic is conferred to the 1DPC biosensor surface by printing three solutions at a concentration of $100 \mu \mathrm{g} / \mathrm{mL}$ in PBS $1 \mathrm{x}$ of three different species of gamma-immunoglobulin (Capture-IgG) over three isolated regions of interest (ROI, Fig. 2a). In particular, onto the three ROIs, we immobilized two antibodies to HER2, e.g. W6/300G9 (ROI 1) and Herceptin (ROI 2). A third antibody to Human Leukocyte antigens (HLA) named L31 (ROI 3) was used as a control of irrelevant specificity. To avoid coalescence between the printed solutions, drops containing a total volume of $10 \mathrm{~nL}$ of solution are printed onto each ROI. The ROIs are separated by a pitch of $1.25 \mathrm{~mm}$. After the Capture-IgG incubation, $45 \mathrm{~min}$ at $+4^{\circ} \mathrm{C}$, the surface is rinsed pipetting Phosphate Buffered Saline solution (PBS 1x) and the microfluidics is mechanically coupled to the biochip.

The first solution injected (Fig.2b I) is BSA $[1 \mathrm{mg} / \mathrm{mL}]$ to passivate the 1DPC surface. The concentrated solution saturates the remaining free glutaraldehyde binding sites preventing undesired nonspecific signals over the ROIs. Whereas, in the formerly dry areas, the BSA binds granting the possibility of setting control regions to track the potential non-specific molecular interactions. The immunoassay performed in this work is a sandwich type. Then, after the injection of a solution of HER2 [ $1 \mathrm{ng} / \mathrm{mL}]$ in skim milk (SM) with a final matrix complexity of $1 \mathrm{mg} / \mathrm{mL}$ (Fig.2b II), fluorescence background is recorded (Fig.2b III). The last step is the injection of a Detection-IgG* labelled with $\operatorname{Qdot}^{\mathrm{TM}} 655[10 \mu \mathrm{g} / \mathrm{mL}]$ in PBS 1x. Being affine for a different epitope of the HER2, the Detection-IgG* brings the fluorophore selectively in those regions of the surface where the analyte has been recognized and captured.

\section{Results}

The focus of this work is to set-up a multiplexed bioassay combining Label-Free and Fluorescence investigation. The Label-Free analysis confirms the successful binding of the Capture-IgG onto each ROI. It can be seen in Fig.2(a) inside the boxed regions where the resonance angle of excitation of the BSW is blue shifted compared to the neighbouring areas. This resonance shift can be translated in surface molecular density by the De Feijter's formula [10] obtaining a mean value of $1173 \pm 77 \mathrm{pg} / \mathrm{mm}^{2}$ of Capture-IgGs.

The recorded sensorgram starts with BSA injection (Fig. 2b I) and clearly shows the difference between the control areas (black, blue, green and purple lines) and the ROIs (red, pink and dark blue lines). This discrepancy is entirely due to the occupancy rate of the glutaraldehyde binding sites. They are mostly saturated by the CaptureIgGs onto the ROIs and completely receptive onto the regions designed to work as reference.

The second resonance shift (Fig. 2b II) is relative to the injection of the skim milk solution containing HER2 [1 $\mathrm{ng} / \mathrm{mL}]$. All the ROIs and the control regions are characterized by the same residual resonance shift, that can be read as the difference between the angular 
resonance position before the biomarker solution injection and after the following PBS 1x washing step.

The last section of the sensorgrams shows the response of the biochip to the injection of a solution of Detection-IgG* labelled with Qdot ${ }^{\mathrm{TM}} 655$ (Fig. 2b IV) and the subsequent washing step with PBS 1x. As in the previous step, the same signal is recorded from all the differently bio-functionalized areas. Therefore, the LabelFree signal analysis gives no information about the interaction between the biosensor surface and the solutions of the biomarker in complex matrix and the Detection-IgG* in PBS 1x.
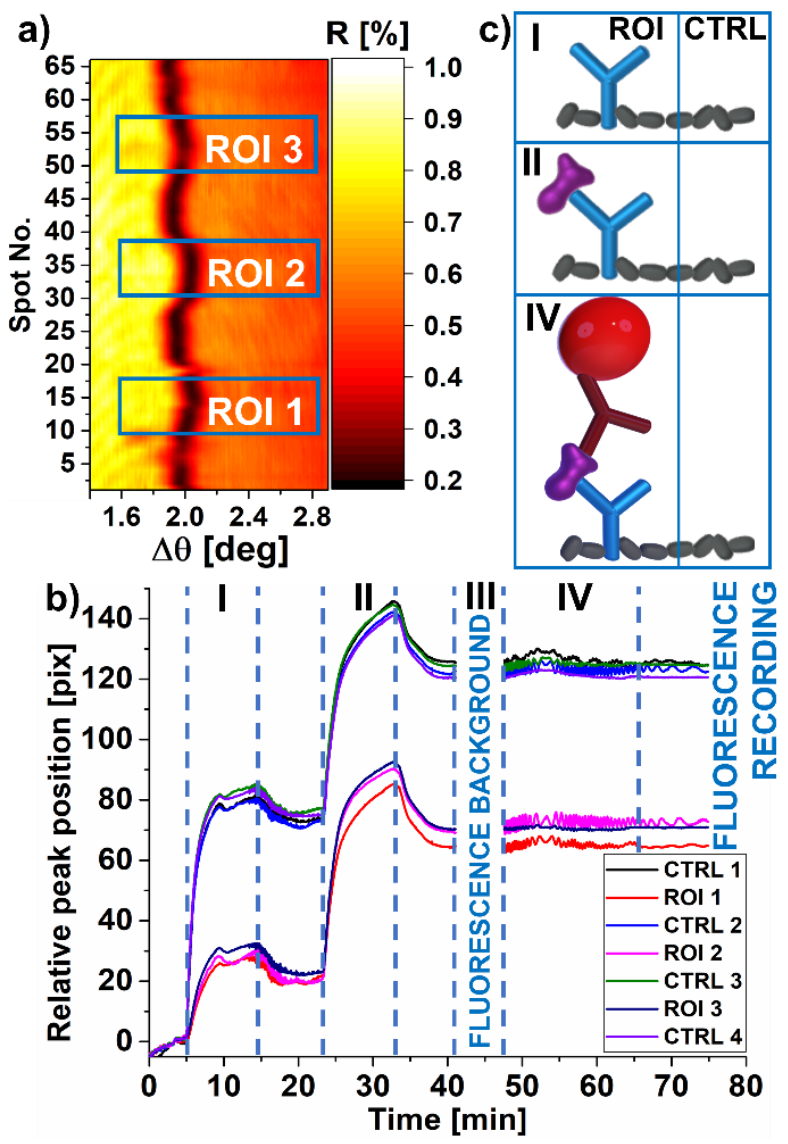

Fig. 2 - In (a) the reflectance angular spectral map along the biochip investigated area is reported. The regions where the three species of antibodies are immobilized are clearly visible. In (b) the real time sensorgrams recorded in Label-Free mode show how the refractive index of the bio-functionalized surface changes when each solution is injected: BSA (I), HER2 in skim milk (II), Detection-IgG* (IV). A surface washing step with PBS $1 \mathrm{x}$ follows each injection. Red, pink and dark blue lines refer to ROIs (1,2 and 3 respectively), while black, blue, green and purple lines refer to control regions (listed as CTRL in (c)).

More information about the last step can be extracted from the Fluorescence signal analysis. To take into account signal aberration due to either stray light from the excitation or fluorescence of the 1DPC, the fluorescence background signal is collected and quantified before the labelled Detection-IgG* (Fig. 2a III) injection. Subtracting this background to the total fluorescence signal collected at the end of the biorecognition protocol, the emitted fluorescence spectral map reported in Fig. 3a is obtained.
In this map the three ROIs are clearly visible where the bioassay ended characterized by different yields (black, blue and green in Fig. 3a-c) and the neighbouring areas used as reference (red in Fig. 3a-c).

From the quantitative analysis of the collected fluorescence (Fig. 3c), one can discriminate the specific (ROI No. 1 and 2) from the non-specific (ROI No. 3) immunoglobulin type. Moreover, the affinity towards the biomarker of the antibody Anti-HER2 (W6/300G9), immobilized onto the ROI No. 1 (black in Fig. 3a-c), is comparable to the affinity shown by the Herceptin (blue in Fig. 3a-c), immobilized onto ROI No. 2.
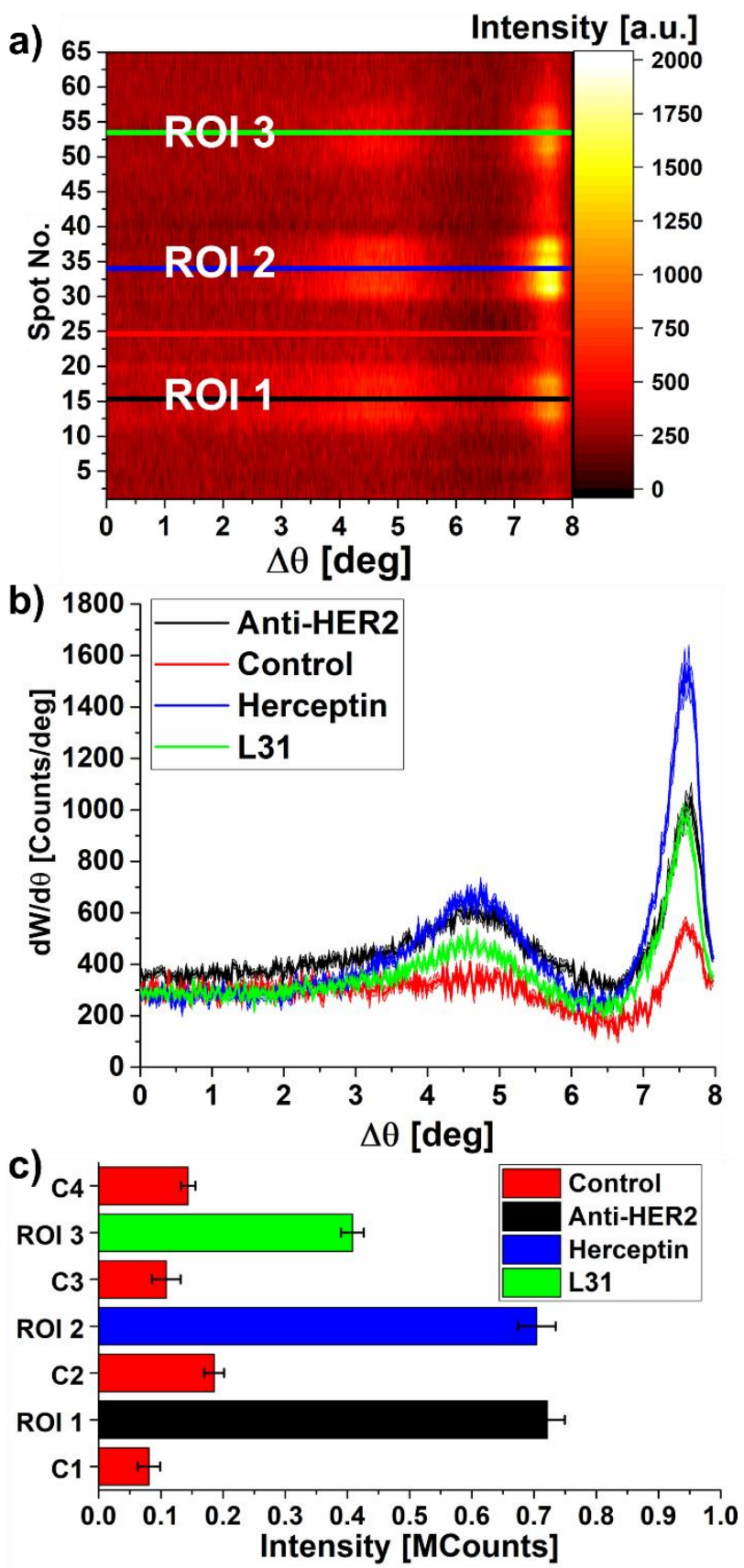

Fig. 3 - In the fluorescence spectral map (a) recorded over the investigated area the three brighter regions correspond to the three ROIs. (b) shows the Fluorescence angular spectra extracted from map (a): black, blue and green spectra correspond to ROI No. 1,2 and 3 respectively, while the red spectrum is extracted from a point of control region No. 2 (listed as C2 
in (c)). The bar plot in (c) illustrates the results including the control signal recorded from the areas between the ROIs.

\section{Conclusions}

In this work we presented a multiplexed optical biosensor based on 1DPC to detect the HER2 biomarker in complex biological matrix. Three different species of immunoglobulins were successfully immobilized onto the biochip surface, achieving a molecular probe density of $1173 \pm 77 \mathrm{pg} / \mathrm{mm}^{2}$ onto each region of interest. The direct comparison of the affinity of the three immunoglobulins for the same antigen was performed in parallel. To take into account of totally non-specific binding events, control areas in which bovine serum albumin was immobilized, were designed. This resulted in a comparable affinity of the two HER2-specific immunoglobulins (W6/300G9 and Herceptin, that is a widely used, well-known therapeutic agent). Furthermore, subtracting the fluorescence signal recorded from the control areas, it turns out that the affinity of the specific immunoglobulins is twice the affinity of the non-specific antibody (L31) towards the same biomarker (Fig. 3c) even at low concentration $(1 \mathrm{ng} / \mathrm{mL})$.

As described herein, combining the two reading modes (Label-Free and Fluorescent), it is possible to discriminate the affinity level of two distinct antibodies to HER2 from the affinity of a non-specific antibody. Furthermore, we proved that the previously demonstrated biosensor performances are not undermined by the integration of three different antibodies and an inert protein onto the same biochip surface. This represents a technical improvement of our previous works opening an integration route and expanding the applicability of an already robust optical biosensor [5].

\section{References}

1. Delfino C.S.C., Perez Cordovés A.I., Souza Jr F.G., Res. Dev. Material. Sci., 7 (2018)

2. Vollmer F., Arnold S., Nat. Methods, 5 (2008)

3. Danz N., Sinibaldi A., Michelotti F., Descrovi E., Munzert P., Shultz U., Sonntag F., Biomed. Tech., 57 (2012)

4. Rizzo R, Alvaro M, Danz N, Napione L, Descrovi E, Schmieder S, et al. Biomed Opt. Express., 9 (2018)

5. Sinibaldi A., Doricchi A., Pileri T., Allegretti M., Danz M., Munzert P., Giordani E., Giacomini P., Michelotti F., Anal. Bioanal. Chem., 412 (2020).

6. Dawood S., Broglio K., Buzdar A.U., Hortobagyi G.N., Giordano S.H., J. Clin. Oncol., 28 (2010)

7. Meric-Bernstam F., Hung M.C., Clinical Cancer Research, 12 (2006)

8. Munzert P, Danz N, Sinibaldi A, Michelotti F., Surf. Coat. Technol., 314 (2017)

9. Gunda N. S. K., Singh M., Norman L., Kaur K., Mitra S.K., Appl. Surf. Sci., 305 (2014)

10. DeFeijter J.A., Benjamins J. and Veer F.A., Biopolymers, 17,1759-1772 (1978) 